\title{
Knowing when to stop antibiotic therapy
}

\section{Empirical antibiotic therapy that turns out to be unnecessary, on review, can (and should) be stopped immediately}

\begin{abstract}
fter 50 years of widespread antibiotic use, we have reached the point where experts are seriously predicting "a postantibiotic era" and the World Health Organization has declared antibiotic resistance "a threat to global security". ${ }^{1}$ No one can doubt the enormous benefits of antibiotics in curing or preventing serious sequelae of infections that were once the main causes of death and chronic illness, and enabling modern medical therapies that involve significant immune suppression.
\end{abstract}

These benefits are dramatic, and toxic side effects are apparently few. This makes it tempting - even now, when we know the risks - to prescribe antibiotics empirically at the first hint of infection, even viral infection, ${ }^{2}$ lest it progress to serious sepsis (and potential medicolegal or professional embarrassment ${ }^{3}$ ). Although unnecessary antibiotic use is sometimes driven by patients' expectations, they can be modified by public education. ${ }^{4}$

During the first 30 years of the antibiotic era, the release of each new antibiotic was almost always followed by the emergence of resistance in some previously susceptible bacteria, but there were always new antibiotics in the pipeline, until recently. Now the pipeline has dried up and the incidence and spectrum of resistance among most common pathogens have reached alarming levels. ${ }^{1}$ How have we come to this point, and what can we do to avoid the "end of the antibiotic era"?

\section{How can we improve our use of antibiotics?}

We still argue about how to optimise antibiotic use, but there are some (more or less) undisputed facts:

- the incidence of antibiotic resistance is, broadly, proportional to the total amount of antibiotics used, ${ }^{5}$ notwithstanding many confounding variables;

- individual antibiotic exposure rapidly alters normal gut microflora, which can take months to recover, risking overgrowth or acquisition of (and, potentially, infection with) multiresistant bacteria, Clostridium difficile or yeasts and spread to hospital, household or nursing home contacts ${ }^{6}-$ and the broader the spectrum and the longer the course, the greater the risk;

- infections with antibiotic-resistant bacteria are more difficult to treat and are associated with higher mortality - antimicrobial resistance is

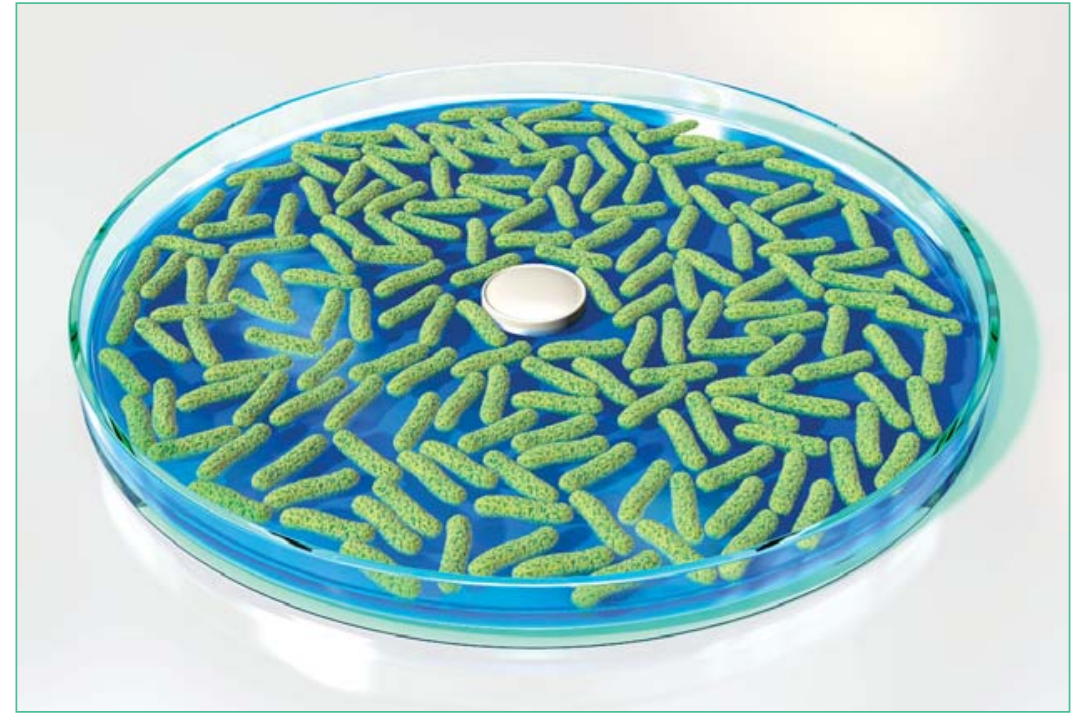

estimated to cost the United States health system US\$21-34 billion per annum; ${ }^{1}$ and

- all antibiotics have some specific adverse side effects such as allergy (or, rarely, anaphylaxis) or dose-related haematological, gastrointestinal, renal or hepatic toxicity.

Surveys of antibiotic use in hospital and community settings show that a third to a half of all prescriptions are discordant with widely available antibiotic guidelines. ${ }^{78}$ Individual decisions to prescribe are often driven by the prescriber's experience, confidence and tolerance of risk, rather than by objective clinical indications. ${ }^{2}$ Antimicrobial stewardship programs are designed to support and share responsibility for logical, evidence-based antibiotic prescribing decisions in the context of inevitable clinical uncertainty, and they can reduce unnecessary - and overall antibiotic use, without adverse patient outcomes.,10

\section{"There is a common misconception that resistance will emerge if a prescribed antibiotic course is not completed"}

In seriously ill patients with suspected bacterial sepsis, initial empirical therapy often means high-dose, broad-spectrum "cover", justified by evidence that the mortality increases rapidly with every hour's delay in

Gwendolyn L Gilbert MD, FRACP, MBioethics

Marie Bashir Institute for Infectious Diseases and Biosecurity, University of Sydney, Sydney, NSW.

lyn.gilbert@ sydney.edu.au

doi: 10.5694/mjal4.01201 starting effective therapy. ${ }^{11}$ For example, recommended empirical therapy for patients with neutropenia who develop fever is to give piperacillin-tazobactam or a fourth-generation cephalosporin..$^{12}$ The need for immediate, effective therapy in severe sepsis is often extrapolated to milder (suspected) infections, with non-specific symptoms, for which therapy may not be necessary or could be delayed until test results are available to guide it. 
Whether to treat and the appropriate choice of empirical therapy are not straightforward decisions, even with the help of prescribing guidelines. However, starting empirical therapy does not mean the patient is committed to a fixed treatment course. Too often, initial therapy is continued without review, even when diagnostic tests indicate an alternative diagnosis (non-infective condition or viral infection) for which no antibiotic is needed or a narrower spectrum agent would suffice. For example, Streptococcus pneumoniae isolated from a blood culture from a patient with severe community-acquired pneumonia is an indication to change from commonly prescribed empirical therapy - ceftriaxone plus azithromycin to benzylpenicillin alone. ${ }^{12}$

\section{Duration of treatment and resistance}

There is a common misconception that resistance will emerge if a prescribed antibiotic course is not completed. Premature cessation of antibiotic therapy will not increase the risk that resistance will emerge. For most infections, the recommended duration of therapy (5-14 days, depending on syndrome) is based on expert opinion and convention, rather than solid evidence. However, for many syndromes associated with bacteraemia, there is no difference in outcome when shorter courses are used..$^{13,14}$ In practice the optimal duration of therapy depends on clinical syndrome, the causative organism, whether source control is possible and the patient's response to therapy. ${ }^{14}$ For example, only 3-5 days of treatment is needed for meningococcal meningitis, compared with 10-14 days for pneumococcal meningitis. ${ }^{12}$ Additional studies are needed to validate shorter courses of antibiotic therapy for many other infections.

Resistance is much more likely to occur with long antibiotic courses, which are rarely indicated except when the site of infection is relatively inaccessible (in biofilm in sites such as a cardiac valve or foreign body or in an abscess); these infections often cannot be cured without surgical removal of the source or drainage of pus. There is no risk - and every advantage - in stopping a course of an antibiotic immediately a bacterial infection has been excluded or is unlikely; and minimal risk if signs and symptoms of a mild infection have resolved.

Competing interests: No relevant disclosures.

Provenance: Commissioned; externally peer reviewed. 
1 World Health Organization. Antimicrobial resistance: global report on surveillance 2014. Geneva: WHO, 2014. http://www. who.int/drugresistance/documents/surveillancereport/en (accessed Oct 2014).

2 Gonzales R, Steiner JF, Sande MA. Antibiotic prescribing for adults with colds, upper respiratory tract infections, and bronchitis by ambulatory care physicians. JAMA 1997; 278: 901-904.

3 Broom A, Broom J, Kirby E. Cultures of resistance? A Bourdieusian analysis of doctors' antibiotic prescribing. Soc Sci Med 2014; 110: 81-88.

4 Wutzke SE, Artist MA, Kehoe LA, et al. Evaluation of a national programme to reduce inappropriate use of antibiotics for upper respiratory tract infections: effects on consumer awareness, beliefs, attitudes and behaviour in Australia. Health Promot Int 2007; 22: 53-64.

5 Goossens H, Ferech M, Vander Stichele R, Elseviers M; ESAC Project Group. Outpatient antibiotic use in Europe and association with resistance: a cross-national database study. Lancet 2005; 365: 579-587.

6 Filius PM, Gyssens IC, Kershof IM, et al. Colonization and resistance dynamics of gram-negative bacteria in patients during and after hospitalization. Antimicrob Agents Chemother 2005; 49: 2879-2886.
7 Zarb P, Amadeo B, Muller A, et al. Identification of targets for quality improvement in antimicrobial prescribing: the web-based ESAC Point Prevalence Survey 2009. J Antimicrob Chemother 2011; 66: 443-449.

8 Stuart RL, Wilson J, Bellaard-Smith E, et al. Antibiotic use and misuse in residential aged care facilities. Intern Med J 2012; 42: $1145-1149$.

9 McGowan JE. Antimicrobial stewardship - the state of the art in 2011: focus on outcome and methods. Infect Control Hosp Epidemiol 2012; 33: 331-337.

10 Davey P, Brown E, Charani E, et al. Interventions to improve antibiotic prescribing practices for hospital inpatients. Cochrane Database Syst Rev 2013; (4): CD003543.

11 Kumar A, Roberts D, Wood KE, et al. Duration of hypotension before initiation of effective antimicrobial therapy is the critical determinant of survival in human septic shock. Crit Care Med 2006; 34: 1589-1596.

12 Antibiotic Expert Groups. Therapeutic guidelines: antibiotic. Version 14. Melbourne: Therapeutic Guidelines Limited, 2010.

13 Havey TC, Fowler RA, Daneman N. Duration of antibiotic therapy for bacteremia: a systematic review and metaanalysis. Crit Care 2011; 15: R267.

14 File TM Jr. Duration and cessation of antimicrobial treatment. J Hosp Med 2012; 7 Suppl 1: S22-S33. 